Document downloaded from:

http://hdl.handle.net/10251/179811

This paper must be cited as:

Simarro, MA.; García Mollá, VM.; Martínez Zaldívar, FJ.; Gonzalez, A. (2020). Low Complexity Near-ML Sphere Decoding based on a MMSE ordering for Generalized Spatial Modulation. IEEE. 1-6. https://doi.org/10.1109/PIMRC48278.2020.9217259

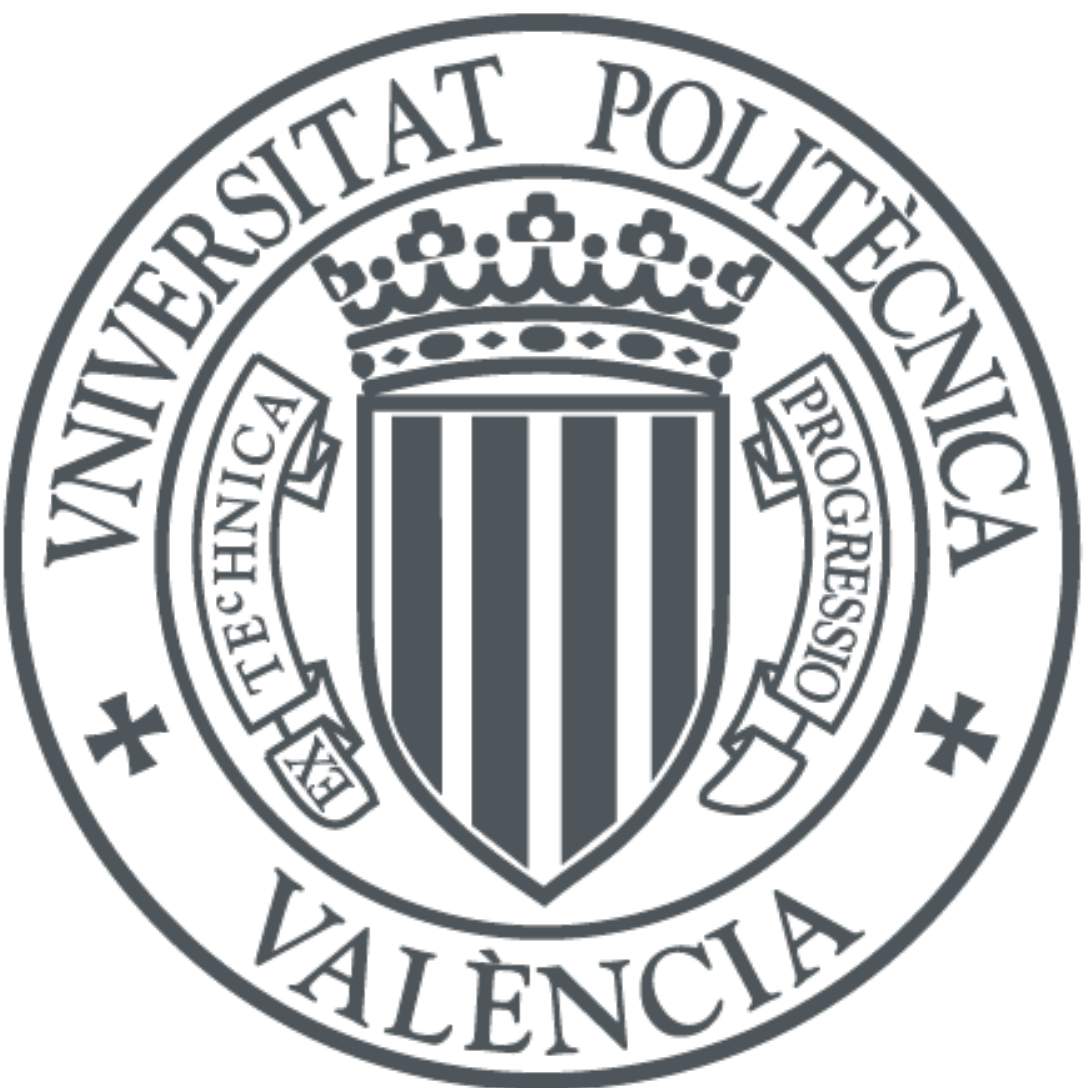

The final publication is available at

https://doi.org/10.1109/PIMRC48278.2020.9217259

Copyright IEEE

Additional Information 


\title{
Low complexity Near-ML Sphere Decoding based on a MMSE ordering for Generalized Spatial Modulation
}

\author{
M.Angeles Simarro*, Victor M. Garcia-Molla ${ }^{\dagger}$, F.J. Martínez-Zaldívar*, Alberto Gonzalez* \\ *ITEAM, Universitat Politècnica de València, Spain. \\ $\{$ mdesiha, fjmartin, agonzal $\} @$ iteam.upv.es \\ ${ }^{\dagger}$ DSIC, Universitat Politècnica de València, Spain. \\ $\{$ vgarcia $\} @$ dsic.upv.es
}

\begin{abstract}
Generalized Spatial Modulation (GSM) is a transmission technique used in wireless communications in which only part of the transmitter antennas are activated during each time signaling period. A low complexity Sphere Decoding (SD) algorithm to achieve maximum likelihood (ML) detection has recently been proposed by using subproblem partitions, sorting preprocessing and radius updating. However, the ordering method has a serious limitation when the number of activated antennas is equal to the number of received antennas. Therefore, alternative sorting methods are studied in the present paper. In addition, the computational cost of the ML algorithm can be high when the system sizes increases. In this paper a suboptimal version is proposed where only the first $\mathrm{L}$ SD subproblems are carried out. The results show that the proposed algorithm achieves near optimal performance at lower computational cost than ML algorithms.
\end{abstract}

Index Terms-Complexity, GSM, MIMO, performance, Signal Detection.

\section{INTRODUCTION}

Multiple-Input Multiple-Output (MIMO) systems [1], and in particular Massive MIMO systems, have gained significant research attention lately because they provide meaningful enhancements in the transmission rate and capacity of communications systems. Recently, an 8 MIMO system has been standardized in the fourth-generation (4G) mobile communication systems and it is expected that massive MIMO with a large number of antennas will be standardized in fifthgeneration (5G) [2].

Spatial Modulation (SM) [3] is another recent and promising transmission technique where only one transmitter antenna is active at any instant of time. The use of only one active transmitter antenna provides good energy efficiency and alleviates the requirement of multiple transmit RF chains in MIMO systems. However SM limits the spectral efficiency that can be achieved.

In a generalized version of SM called Generalized Spatial Modulation (GSM) systems [4] more than one transmitter antenna is active at the same time, overcoming the drawbacks of SM. Thus, GSM keeps the advantages of SM with respect to the conventional MIMO systems but increasing the system spectral efficiency. Due to the advantages of GSM, it is currently being studied as an emerging challenge for the $5 \mathrm{G}$ [5]. Unfortunately the detection process of GSM becomes more complex since the receiver needs to detect both the transmitted symbols and the subset of antennas chosen for the transmission.

Different strategies have usually been considered in order to recover the information at the receiver side. One strategy is to detect the bits into two stages. In the first stage, the antenna combination (the subset of selected antennas) is determined and then the transmitted signal is detected by solving a smaller MIMO detection scheme. Some examples of suboptimal detectors that apply this strategy can be found in [2] [6] [7]. Otherwise, an extended constellation by considering the transmitted signals from the active antennas and null symbols from the inactive antennas is used in [8] [9] [10].

On the other hand, a suboptimal algorithm called Ordered Block minimum mean squared error (OBMMSE) was proposed in [6] where the detection of the antenna combination and the detection of the symbols are carried out jointly. The OBMMSE can achieve an error rate performance close to the maximal likelihood (ML) performance under certain configuration setups.

Additionally, a recent ML method called Sorting Assisted Successive Sphere Decoding Algorithm (SA-SSDA) was proposed in [11]. This algorithm uses successive applications of Sphere Decoders (SD), one for each valid transmit antenna combination (TAC). This algorithm is an efficient ML detector for GSM. The efficiency is achieved by employing an adjustable decoding radius and an ordering step of the valid TACs. Thereby the combinations with higher reliability are processed first. However, as will be shown later, the ordering method proposed in [11] is not efficient under certain configuration settings.

Motivated by the above, alternative TAC sorting have been evaluated in order to overcome the restriction of the original algorithm proposed in [11]. The ordering methods evaluated through the paper are based in two well known estimators: Zero-Forcing (ZF) [12] and Minimum Mean Square Error (MMSE) [13]. The main goal of this paper is to present a 
suboptimal algorithm called L-first MMSE algorithm. This algorithm employs the MMSE ordering step, which allows to execute only the first L SD subproblems with minimal loss of performance. A suitable L parameter based on the reliability of the detected solution is adjusted during the execution. The results show that the proposed detector can achieve an error rate close to the ML detector, while its complexity is significantly reduced.

The remainder of this paper is organized as follows. In Section II the system model for GSM scheme is described. In Section III we briefly discuss the ML detection problem in GSM and a recent ML method is revised. In Section IV, the L-first proposed algorithm is presented. Computer simulation results are given in Section V. Finally, Conclusions are given in Section VI.

Notation: Upper and lower case boldface letters denote matrices and vectors, respectively. ()$^{H},()^{-1}$ and $\|\cdot\|$ denote the Hermitian transpose, inversion and 2 -norm operation, respectively. $|\cdot|$ denotes the cardinality of a set. $\left(\begin{array}{l}n \\ k\end{array}\right)$ denotes the binomial coefficient, the number of combinations in which $k$ items can be taken from a set of $n$ items.

\section{PRoblem DESCRIPTION}

Consider a GSM system over a MIMO channel using $n_{T}$ transmit antennas and $n_{R}$ receive antennas. In the GSM system, only $n_{A}\left(2 \leq n_{A} \leq n_{T}\right)$ transmitter antennas are activated at each period of time. Consequently, the total number of possible TAC is $\left(\begin{array}{l}n_{T} \\ n_{A}\end{array}\right)$. Among these TACs some of them are considered as valid TACs. If $n_{b}$ bits of information are used to select the valid TAC, then $N_{c}=2^{n_{b}}$ valid TACs are chosen from the total TACs. Thus, a TAC index, $k \in \theta=\left\{1,2, \cdots, N_{c}\right\}$ is therefore associated with a set of $n_{A}$ active antenna indices $\left\{i_{1}, i_{2}, \cdots, i_{n_{A}}\right\}$.

In addition, the $n_{A}$ activated transmit antennas are used to transmit $n_{A}$ independent $M$-ary modulation symbols belonging to the $\Omega$ constellation of size $|\Omega|=M$, and hence to convey additional $n_{A} \log _{2}(M)$ bits. Thereby, the bits to be transmitted are grouped in blocks of $n_{A} \log _{2}(M)+\log _{2}\left(N_{c}\right)$. The first $n_{A} \log _{2}(M)$ bits are mapped into a symbol vector $\mathbf{s}=\left[s_{1}, \cdots, s_{n_{A}}\right]$. The remaining $n_{b}$ bits are used to select the valid TAC.

Let $\mathbf{H} \in \mathbb{C}^{n_{R} \times n_{T}}$ be the MIMO channel matrix. Consequently, the received signal $\mathbf{y} \in \mathbb{C}^{n_{R} \times 1}$ can be described as $\mathbf{y}=\mathbf{H x}+\mathbf{v}$, where $\mathbf{x}=\left[\cdots, 0, s_{1}, 0, \cdots, s_{2}, 0, \cdots, s_{n_{A}}\right.$, $0, \cdots]^{T}$ denotes the transmitted symbol vector, and $\mathbf{v} \in$ $\mathbb{C}^{n_{R} \times n_{T}}$ denotes an additive white-Gaussian noise (AWGN) with variance $\sigma^{2}$. If the transmission is carried out through the $k^{\text {th }}$ valid TAC, the corresponding channel submatrix can be defined as $\mathbf{H}_{\mathbf{k}} \in \mathbb{C}^{n_{R} \times n_{A}}$. Therefore the received signal vector can be represented as

$$
\mathbf{y}=\mathbf{H}_{\mathbf{k}} \mathbf{s}+\mathbf{v} .
$$

The receiver side has to recover both, the modulated symbol vector $\mathbf{s}$ and the TAC index $k \in \theta$. With this notation, the ML detector for the GSM problem can be described as

$$
(\hat{k}, \hat{\mathbf{s}})=\arg \min _{k \in \theta, \mathbf{s} \in \Omega^{n} A}\left\|\mathbf{y}-\mathbf{H}_{\mathbf{k}} \mathbf{s}\right\|^{2} .
$$

\section{ML DETECTION WITH ADJUSTABLE RADIUS AND ORDERING PREPROCESSING}

Standard ML MIMO detection algorithms cannot be directly applied to GSM problems when $n_{T}>n_{R}$ because it is not possible to obtain the required QR factorization of the channel matrix. Furthermore, these methods have a high computational complexity. However, to compute the ML solution we can decouple the problem into $N_{c}$ subproblems and solve one ML subproblem for each valid TAC as:

$$
\hat{\mathbf{s}}_{\mathbf{k}}=\arg \min _{\mathbf{s} \in \Omega^{n} A}\left\|\mathbf{y}-\mathbf{H}_{\mathbf{k}} \mathbf{s}\right\|^{2} .
$$

The optimal selection of $\hat{k}$ and $\hat{\mathbf{s}}$ will be given by the TAC that gives the minimum Euclidean distance $d_{k}=\left\|\mathbf{y}-\mathbf{H}_{\mathbf{k}} \hat{\mathbf{s}}_{\mathbf{k}}\right\|^{2}$ for $k=1, \cdots, N_{c}$. The Successive Sphere Decoding Algorithm (SSDA) [11] employs one SD to solve (3) for each k, with $k=$ $1, \cdots, N_{c}$. However, the cost of solving $N_{c}$ SD subproblems can be very high. Nevertheless, an adjustable radius and an ordering preprocessing stage can be used in order to reduce the computational complexity. The algorithm that implements these strategies is called SA-SSDA [11].

The first idea proposed in [11] is to reduce the computational cost employing an adjustable radius. In its search for the ML solution, the standard MIMO SD detectors use an initial radius $C_{o}$, which is updated during the detection procedure when a better solution is found. Thus, the search is reduced to a hyper-sphere of radius $C_{o}$. The idea of an adjustable radius is extended for GSM problems. Thus, the best radius obtained in a given TAC can be propagated as the initial radius for the next TAC.

The second idea is to carry out an ordering preprocessing step. The goal of this step is that the detected valid TACs, which have the highest probability of being the correct one, are taken into account in the first places. In this way, the smallest radius will be found in those places, and along with the radius propagation strategy, the computational cost of the whole detection process is considerably reduced.

\section{L-FIRST MMSE DETECTION}

The SA-SSDA ML detector is very efficient with low size GSM problems. However, for larger problems its computational cost becomes unfeasible. The motivation of this paper is to achieve the near-ML performance with reduced complexity, for this purpose we propose a novel detection scheme called Lfirst MMSE. The main idea is to execute a sorting step which puts the correct TAC in the first places. Therefore, the correct TAC is tested on the first solved ML subproblems, resulting in the fact that it is not necessary to test all the $N_{c}$ valid TACs. Hence, we can conclude that a good ordering method is needed that puts the correct TAC in the first places. The second issue to solve is to find a suitable L parameter in order to reduce the complexity while keeping near-ML performance. 


\section{A. Ordering preprocessing}

The ordering method proposed in SA-SSDA algorithm depends on the QR decomposition of the channel submatrices $\mathbf{H}_{\mathbf{k}}$ with $k=1, \cdots, N_{c}$, which can be rewritten as:

$$
\mathbf{H}_{\mathrm{k}}=\mathbf{Q}_{\mathrm{k}} \mathbf{R}_{\mathrm{k}}=\mathbf{Q}_{\mathrm{k}}\left(\begin{array}{c}
\mathbf{R}_{\mathrm{k} 1} \\
0
\end{array}\right)=\left(\begin{array}{ll}
\mathbf{Q}_{\mathrm{k} 1} & \mathbf{Q}_{\mathrm{k} 2}
\end{array}\right)\left(\begin{array}{c}
\mathbf{R}_{\mathrm{k} 1} \\
0
\end{array}\right)
$$

where $\mathbf{R}_{\mathbf{k} 1} \in \mathbb{C}^{n_{A} \times n_{A}}, \mathbf{Q}_{\mathbf{k} \mathbf{1}} \in \mathbb{C}^{n_{R} \times n_{A}}$ and $\mathbf{Q}_{\mathbf{k} \mathbf{2}} \in$ $\mathbb{C}^{n_{R} \times\left(n_{R}-n_{A}\right)}$. The $\mathrm{QR}$ decomposition is performance in $n_{R} \times n_{A}$ size matrices, being $n_{A} \ll n_{T}$. Furthermore, for slow fading channel the $\mathrm{QR}$ decomposition of $\mathbf{H}_{k}$, produces a reduced cost in the total computation.

We can rewrite (2) as

$$
\begin{aligned}
(\hat{k}, \hat{\mathbf{s}})= & \arg \min _{k \in \theta}\left(\left\|\mathbf{Q}_{\mathbf{k} \mathbf{2}}{ }^{H} \mathbf{y}\right\|^{2}+\right. \\
& \left.\min _{\mathbf{s} \in \Omega^{n} A}\left\|\mathbf{Q}_{\mathbf{k} \mathbf{1}}{ }^{H} \mathbf{y}-\mathbf{R}_{\mathbf{k} \mathbf{1}} \mathbf{s}\right\|^{2}\right) .
\end{aligned}
$$

Then, it sorts the valid TACs by ordering the term $\left\|\mathbf{Q}_{\mathbf{k} \mathbf{2}}{ }^{H} \mathbf{y}\right\|^{2}$, which is independent of $\mathbf{s}$. However, when $n_{R}=$ $n_{A}$, this term disappears of (5) and thus it cannot be used to order the TACs. For this reason, two different ordering methods are analyzed as meaningful alternatives. First of all, a simple but effective ordering method, proposed in [7], and based on the ZF estimator is employed. The ZF solution for each valid TAC is given by

$$
\hat{\mathbf{z}}_{\mathbf{k}}=\mathcal{Q}\left(\left(\mathbf{H}_{\mathbf{k}}{ }^{H} \mathbf{H}_{\mathbf{k}}\right)^{-1} \mathbf{H}_{\mathbf{k}}{ }^{H} \mathbf{y}\right)
$$

where $\mathcal{Q}(\cdot)$ gives the nearest constellation symbol. The Euclidean distances of these estimators are computed as $d_{z_{k}}=$ $\left\|\mathbf{y}-\mathbf{H}_{\mathbf{k}} \hat{\mathbf{z}}_{\mathbf{k}}\right\|^{2}$. Then, the valid TACs are sorted according to $d_{z_{k}}$, from the smallest to largest. This method does not have any restrictions. Furthermore, as we will show in the Section $\mathrm{V}$, this ordering method considerably reduces the number of visited nodes by the SD.

However, when the MIMO channel $\mathbf{H}_{\mathbf{k}}$ is ill-conditioned, the ZF estimator suffers from the well-known noise enhancement problem: the noise is amplified along those directions corresponding to small singular values of $\mathbf{H}_{\mathbf{k}}$. Then the MMSE estimator can be used instead. The MMSE solution is given by

$$
\hat{\mathbf{w}}_{\mathbf{k}}=\mathcal{Q}\left(\left(\mathbf{H}_{\mathbf{k}}{ }^{H} \mathbf{H}_{\mathbf{k}}+\sigma^{2} \mathbf{I}\right)^{-1} \mathbf{H}_{\mathbf{k}}{ }^{H} \mathbf{y}\right) .
$$

Thus, the Euclidean distance of the MMSE estimator is computed as $d_{w_{k}}=\left\|\mathbf{y}-\mathbf{H}_{\mathbf{k}} \hat{\mathbf{w}}_{\mathbf{k}}\right\|^{2}$ and then the valid TACs are sorted, from the smallest to largest $d_{w_{k}}$.

In Section V, the sorting operation based on both estimators (ZF and MMSE) has been tested and analyzed under different parameter configurations. The results lead us to employ the MMSE estimator in the sorting step.

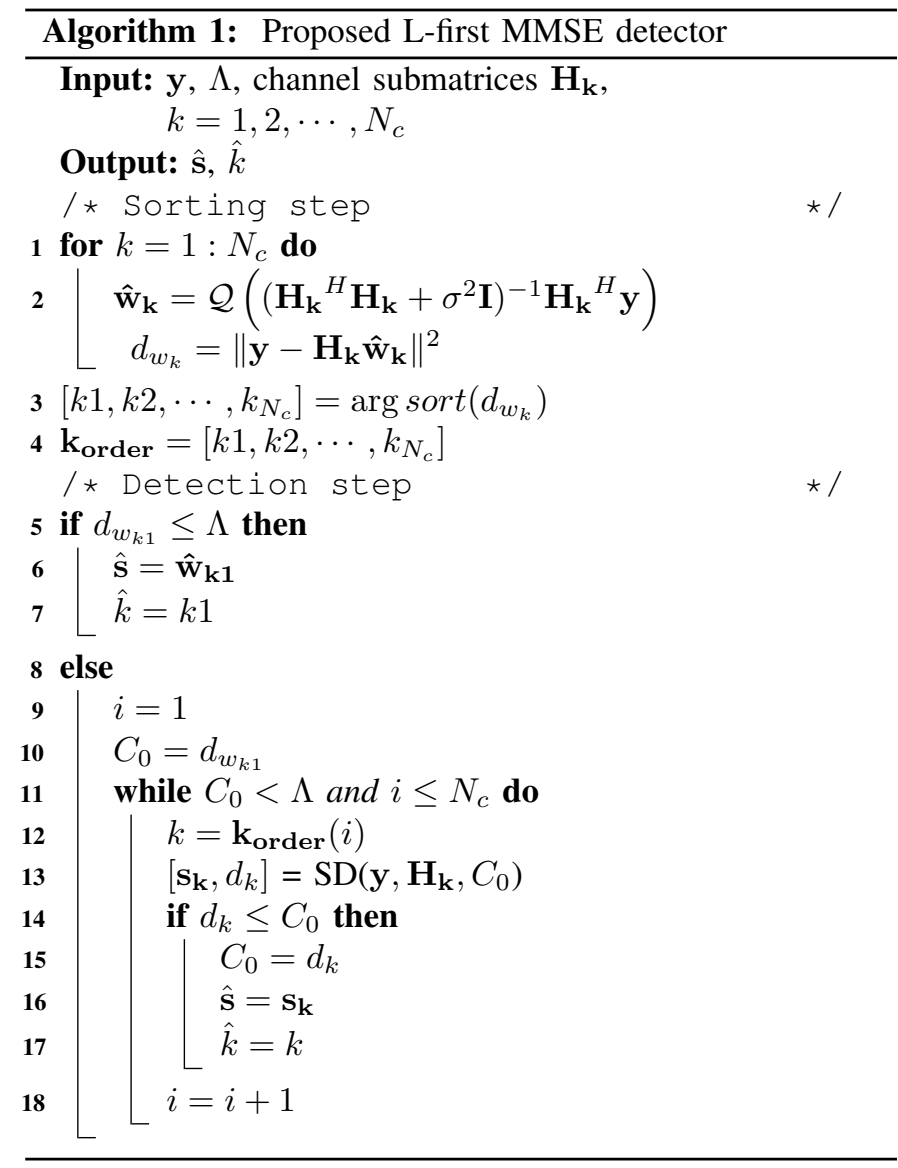

\section{B. Adjustable L value}

The main idea of the proposed algorithm is to execute the sorting step employing the MMSE estimator and after that evaluates only the L-first subproblems of (3) instead of $N_{c}$. Thus, it is important to choose a suitable $\mathrm{L}$ value in order to obtain minimum performance degradation.

For this purpose, we use a threshold $\Lambda$ to guess how many L ML subproblems need to be solved. In this way, $\Lambda$ is a threshold to define the reliability of the detected signal vector. According to [14], $\Lambda$ can be computed as:

$$
\Lambda=n_{R} \sigma^{2}
$$

Thus, when a ML subproblem is solved, in addition to evaluate if the present radius is smaller than the adjustable initial radius $C_{o}$, we also have to judge if the estimated transmit vector is reliable or not according to:

$$
\left\|\mathbf{y}-\mathbf{H}_{\mathbf{k}} \hat{\mathbf{s}}_{\mathbf{k}}\right\|^{2} \leq \Lambda
$$

If the evaluated $k^{t h}$ valid TAC fulfills equation (9), we can discard the rest of valid TACs, thus reducing the computational cost. Thus, the $\mathrm{L}$ value will be variable and adjustable given by $\Lambda$. This procedure is implemented in Algorithm 1 . Furthermore, if after the sorting step, the distance associated with the first MMSE estimator is lower than the threshold $\Lambda$, it is not necesary to execute any SD algorithm. The solution 
will be given by the first MMSE estimator after the sorting step.

\section{SIMULATION RESULTS}

In this section, we present the results obtained by Monte Carlo simulation. The experiments were carried out varying the signal-to-noise ratio (SNR) and employing Rayleigh fading channel with a channel coherence time of 5 transmission periods. Each simulation point has been obtained by averaging the number of over 10000 independent realizations. Two different setups have been chosen for simulation (see Table V).

TABLE I

SETUPS For Computed Simulations

\begin{tabular}{|c|c|c|c|c|}
\hline & $n_{T}$ & $n_{A}$ & $n_{R}$ & $N_{c}$ \\
\hline Setup 1 & 8 & 2 & 8 & 16 \\
\hline Setup 2 & 32 & 6 & 6 & 64 \\
\hline
\end{tabular}

\section{A. Ordering preprocessing Evaluation}

First of all, the different sorting methods have been analyzed. The original sorting step of the SA-SSDA algorithm has been compared with the MMSE and the ZF estimators for the ordering preprocessing, calling the algorithms MMSESSDA and ZF-SSDA, respectively. It is important to highlight that the three sorting methods and the successive SD algorithm achieve the ML BER performance.

The number of visited nodes is widely used to measure the complexity of the SD algorithms, for this reason it has been used to measure the efficiency of the sorting step. That is, the sorting method that places firstly the most reliable TACs achieves the lowest number of visited nodes. Figures 1 and 2 illustrate the average number of visited nodes for the three different sorting methods and the two different setups. It is important to note, that the tree algorithm employs the SSDA algorithm to achieve the optimal solution. The only difference is the preprocessing stage in which three different ordering methods are evaluated. From the results, we can conclude that, depending on the ordering method of the valid TACs different computational cost (evaluated in our case in number of expanded nodes) is achieved. The reason behind these differences in the computational cost is based on the fact that the method which expands lower number of nodes implements a more efficient ordering step. Thus means, this method puts the optimal TAC at the first places. Figure 3 illustrates this claim. This Figure represents the percentage of times when the optimal TAC is placed on the first five evaluated TACs (after the ordering step). Figure 3 shows that the MMSE ordering method sets the valid TAC at the first places with higher probability than the other ordering methods. Thus, the results led us to choose the MMSE ordering method as the better alternative for our L-first algorithm.

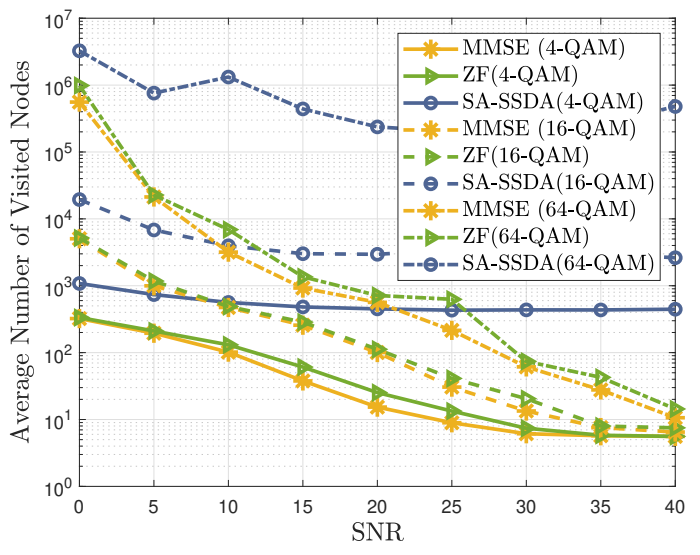

Fig. 1. Complexity comparisons for ML algorithms under a GSM MIMO system with $n_{T}=32, n_{A}=6, n_{R}=6$ and $N_{c}=64$.

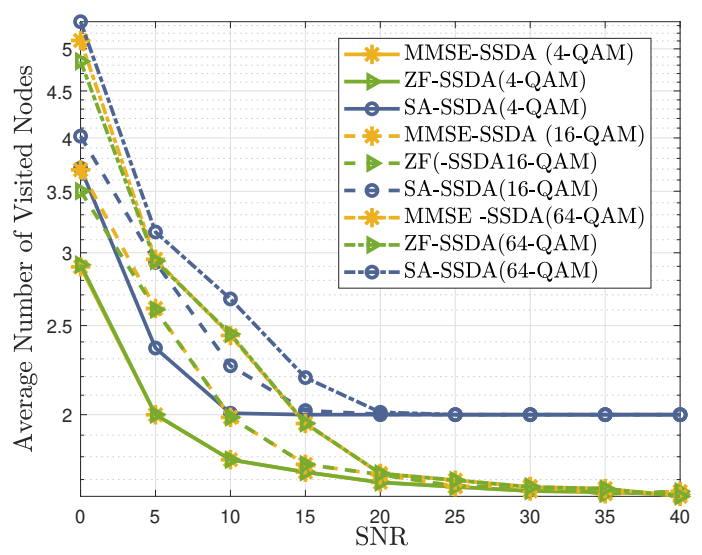

Fig. 2. Complexity comparisons for ML algorithms under a GSM MIMO system with $n_{T}=8, n_{A}=2, n_{R}=8$ and $N_{c}=16$.

\section{B. L-first MMSE detector Evaluation}

In this section the L-first MMSE detector has been compared with the original SA-SSDA algorithm (which achieves ML performance). For a more complete comparison we have also plotted the performance of the popular suboptimal OBMMSE algorithm. The BER curves of the three algorithms are provided in Figures 4 and 5 for the different setups. Figures show that the performance of the proposed L-first MMSE algorithm is close to the ML detector performance. Furthermore, the computational complexities of the L-first MMSE detector and the SA-SSDA algorithm have been analyzed in this section. Specifically, the computational complexity has been measured by the number of visited nodes and also by the total number of flops. The results are shown in Figures 6 and 7 . The proposed algorithm can reduce the complexity by up to several orders of magnitude in most cases. In this sense and in regard to the obtained results, we can confirm that the proposed algorithm can achieve near-ML performance with a considerably reduced complexity. 


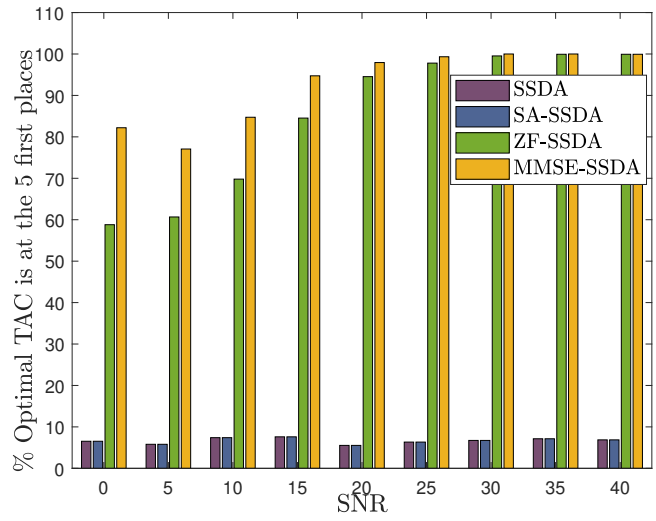

Fig. 3. Percentage of time when the optimal TAC is placed on the first five evaluated TACs (after the ordering step) in a GSM MIMO system with $n_{T}=32, n_{A}=6, n_{R}=6$ and $N_{c}=64$.

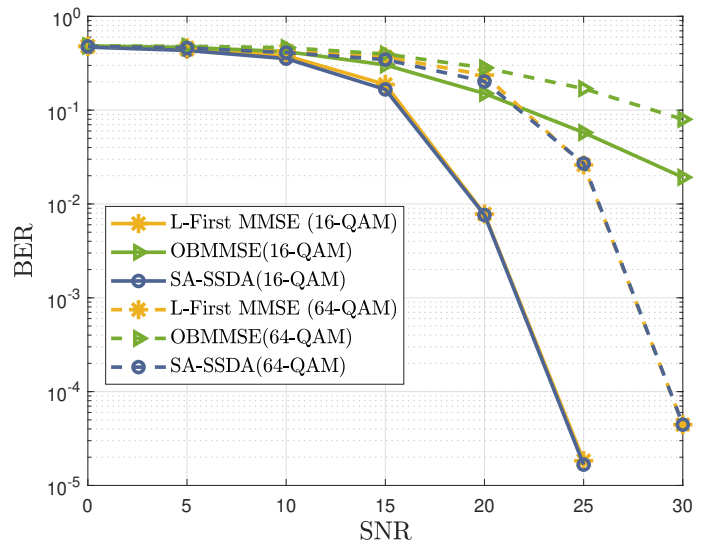

Fig. 4. BER curves for a GSM MIMO system with $n_{T}=32, n_{A}=6$, $n_{R}=6$ and $N_{c}=64$.

\section{CONCLUSIONS}

This paper compares and evaluates different ordering methods for the valid TACs. Furthermore, using best sorting method a novel L-first MMSE detection algorithm is proposed. This algorithm only needs to solve the L first ML TAC subproblems. Compared to the faster ML algorithm, the simulation curves show that the proposed algorithm provides a near-ML detection performance by reducing the overall complexity.

\section{ACKNOWLEDGMENT}

This work has been partially supported by Spanish Ministry of Science, Innovation and Universities and by European Union through grant RTI2018-098085BC41 (MCUI/AEI/FEDER), by GVA through PROMETEO/2019/109 and by Catedra Telefonica-UPV through SSENCE project.

\section{REFERENCES}

[1] D. Gesbert, M. Shafi, D. S. Shiu, P. J. Smith, and A. Naguib, "From theory to practice: An overview of MIMO space-time coded wireless

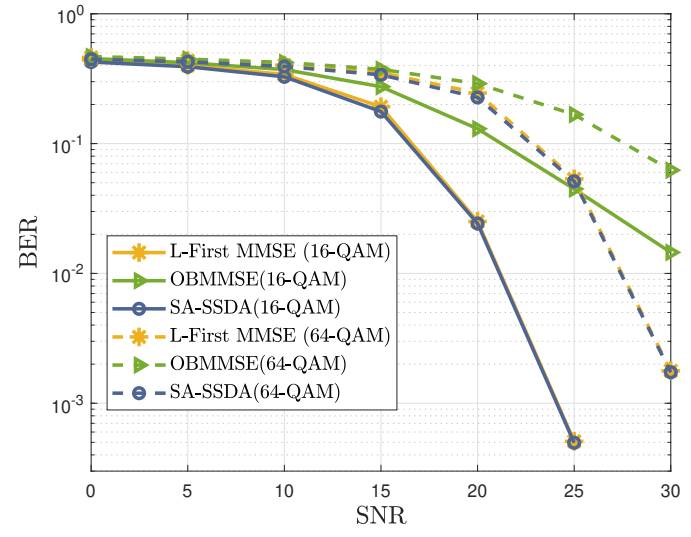

Fig. 5. BER curves for a GSM MIMO system with $n_{T}=8, n_{A}=2$, $n_{R}=8$ and $N_{c}=16$.
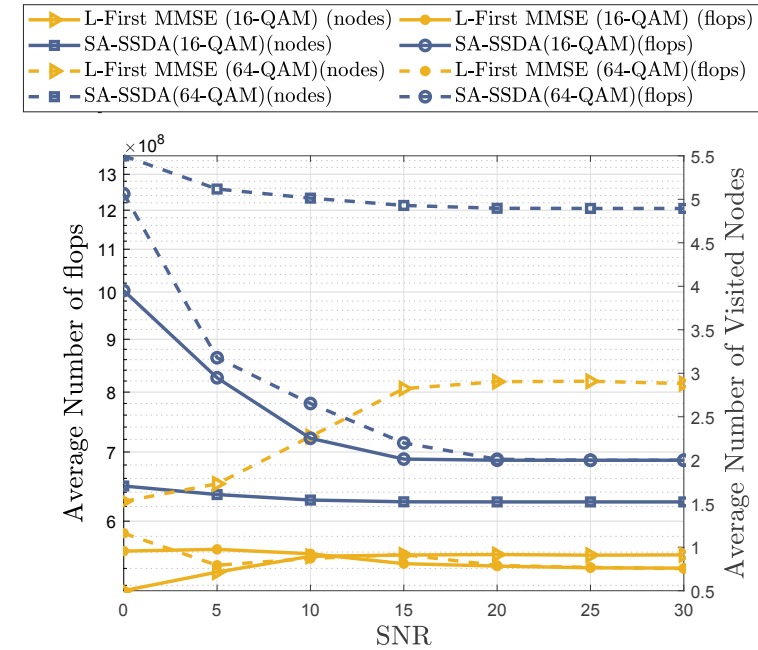

Fig. 6. Complexity comparisons in terms of average of visited nodes and flops for a GSM MIMO system with $n_{T}=8, n_{A}=2, n_{R}=8$ and $N_{c}=16$.

systems," IEEE Journal on Selected areas in communications, vol. 21, no. 3, pp. 281-302, 2003.

[2] N. Hassan and X. Fernando, "Massive MIMO wireless networks: An overview," Electronics, vol. 6, no. 3, p. 63, 2017.

[3] R. Y. Mesleh, H. Haas, S. Sinanovic, C. W. Ahn, and S. Yun, "Spatial modulation," IEEE Transactions on vehicular technology, vol. 57, no. 4 pp. 2228-2241, 2008.

[4] J. Wang, S. Jia, and J. Song, "Generalised spatial modulation system with multiple active transmit antennas and low complexity detection scheme," IEEE Transactions on Wireless Communications, vol. 11, no. 4, pp. 1605-1615, 2012.

[5] P. Patcharamaneepakorn, S. Wu, C.-X. Wang, M. M. Alwakeel, X. Ge, M. Di Renzo et al., "Spectral, energy, and economic efficiency of $5 \mathrm{~g}$ multicell massive mimo systems with generalized spatial modulation," IEEE Transactions on Vehicular Technology, vol. 65, no. 12, pp. 97159731, 2016.

[6] Y. Xiao, Z. Yang, L. Dan, P. Yang, L. Yin, and W. Xiang, "Lowcomplexity signal detection for generalized spatial modulation," IEEE Communications Letters, vol. 18, no. 3, pp. 403-406, 2014.

[7] G. Altın and M. E. Çelebi, "A simple low-complexity algorithm for generalized spatial modulation," AEU-International Journal of Electronics and Communications, vol. 97, pp. 63-67, 2018.

[8] Y. Jiang, Y. Lan, S. He, J. Li, and Z. Jiang, "Improved low-complexity sphere decoding for generalized spatial modulation," IEEE Communica- 

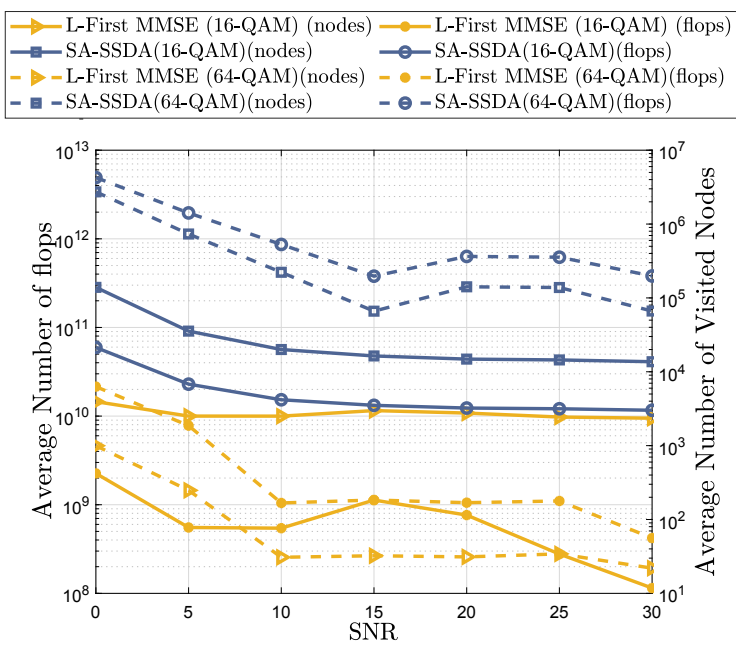

Fig. 7. Complexity comparisons in terms of average of visited nodes and flops for a GSM MIMO system with $n_{T}=32, n_{A}=6, n_{R}=6$ and $N_{c}=64$.

tions Letters, vol. 22, no. 6, pp. 1164-1167, 2018.

[9] L. Xiao, P. Yang, Y. Xiao, S. Fan, M. Di Renzo, W. Xiang, and S. Li, "Efficient compressive sensing detectors for generalized spatial modulation systems," IEEE Transactions on Vehicular Technology, vol. 66, no. 2, pp. 1284-1298, 2016.

[10] T. Q. Tran, S. Sugiura, and K. Lee, "Ordering-and partitioning-aided sphere decoding for generalized spatial modulation," IEEE Transactions on Vehicular Technology, vol. 67, no. 10, pp. 10 087-10091, 2018.

[11] T.-H. Liu, C.-E. Chen, and C.-H. Liu, "Fast Maximum Likelihood Detection of the Generalized Spatially Modulated Signals Using Successive Sphere Decoding Algorithms," IEEE Communications Letters, vol. 23, no. 4, pp. 656-659, 2019.

[12] C. Wang, E. K. Au, R. D. Murch, W. H. Mow, R. S. Cheng, and V. Lau, "On the performance of the mimo zero-forcing receiver in the presence of channel estimation error," IEEE transactions on wireless communications, vol. 6, no. 3, pp. 805-810, 2007.

[13] N. Kim, Y. Lee, and H. Park, "Performance analysis of mimo system with linear mmse receiver," IEEE Transactions on Wireless Communications, vol. 7, no. 11, pp. 4474-4478, 2008.

[14] F. Wang, Y. Xiong, and X. Yang, "Approximate ML detection based on MMSE for MIMO systems," PIERS online, vol. 3, no. 4, pp. 475-480, 2007. 